\title{
La frontière des « écrits bruts » : pour une dé-limitation du chant de la folie
}

\author{
Pauline Goutain \\ Université Carleton
}

Le terme "écrits bruts» est apparu dans la continuité du concept d' « art brut ». Ce terme, dû en 1945 au peintre français Jean Dubuffet, visait à remettre en question la définition traditionnelle de l'art. Tout comme l'« art brut» avait été conçu par Dubuffet en tant qu'art « anti-culturel »1, les « écrits bruts » ont été définis, une trentaine d'années plus tard, par opposition

\footnotetext{
${ }^{1}$ Le texte de Dubuffet L'Art brut préféré aux arts culturels (1949) fait office de manifeste quant à la définition de l'«art brut» et positionne clairement ce concept contre celui de « culture».
} 
à la littérature traditionnelle. Dans l'ouvrage-manifeste Les Écrits bruts (Thévoz, 1979), marginalité et non-communication sont mises en avant ainsi que l'idée de non-érudition. La notion de folie joue également un rôle majeur dans la définition de cette catégorie. En effet, ces textes proviennent majoritairement d'hôpitaux psychiatriques et mettent en scène les délires, les utopies, les réclamations de personnes internées.

Les enjeux de la «frontière » dans les « écrits bruts » sont multiples. Si le mot «frontière » désigne en premier lieu les limites d'un territoire géographique, il permet également de déterminer le champ d'application d'un concept et de le positionner par rapport à d'autres notions. La frontière peut être matérielle ou figurée, contribuer à l'organisation d'un espace concret ou à la structuration de l'espace mental. La notion de "frontière " peut aussi être conçue comme une ligne de séparation impliquant un rapport de force ou bien une interface permettant le dialogue et l'échange. Ces différents aspects de la frontière permettent d'aborder les "écrits bruts » sous différents angles: des limites conceptuelles de cette catégorie aux frontières concrètes de ce type d'écriture - à savoir son contexte de création - en passant par la frontière du support - cette ligne qui délimite un champ d'expression - et la question du signe - interface plus ou moins transparente où raison et folie se réconcilient.

D'autre part, la question de la frontière au sein des "écrits bruts» amène à s'interroger plus largement sur le processus de reconnaissance et sur le rôle de l'écriture en soi, au-delà du champ strict de la littérature. Tout d'abord, dans le discours avant-gardiste associé aux " écrits bruts », la notion de frontière, par le biais des métaphores spatiales, semble prendre 
une dimension stratégique. La limite dessinée entre "écrits bruts » et littérature tend à conférer une valeur subversive à ces textes et à en faire un outil critique allant à l'encontre de la norme et du monde de la culture. La frontière concrète des « écrits bruts » - les murs de l'asile - vient, de plus, remettre en question le contexte qui assure à l'écriture son statut de littérature. Enfin, la frontière du sens créée par une langue affolée nous invite à reconsidérer l'écriture non plus comme un simple véhicule de sens mais comme un acte poétique, inventif et existentiel.

Ainsi, nous chercherons à explorer les différentes strates de la notion de frontière afin de mettre au jour les différentes perspectives que le concept d'«écrits bruts» ouvre sur l'écriture, la littérature, voire l'art.

Les « écrits bruts » au sein de l'«art brut»: une question de catégories?

\section{Littérature et arts visuels : deux nations étrangères?}

Dans l'histoire de la pensée occidentale, le domaine de l'esprit a souvent eu la part belle sur celui de la main et du faire. Les arts libéraux - par opposition aux arts mécaniques - comptaient ainsi au Moyen Âge seulement les productions de la pensée, l'« ensemble des choses écrites» (Suberville, 1948, p. 9) auxquelles on reconnaissait une valeur esthétique et un contenu érudit. Ce n'est qu'à la Renaissance que peinture, dessin, sculpture et architecture intègrent cette catégorie. Les BeauxArts sont ainsi devenus une catégorie relevant de l'esthétique au même titre que les Belles-Lettres. Toutefois, si ces deux 
disciplines se sont vu attribuer le privilège de relever de l'intelligence humaine et du Beau, elles ont été séparées en deux univers différents - l'un qui a trait aux mots; l'autre, aux formes et couleurs. La création des Académies (XVIIe et XVIIIe siècles) a contribué à officialiser cette frontière. En France, notamment, la fondation de l'Académie française, chargée du domaine littéraire et de la langue française (1635), est allée de pair avec la création de l'Académie royale de peintures et de sculptures, chargée des Beaux-Arts (1648). Ces deux institutions se sont maintenues dans le temps et régissent toujours la distinction faite entre langages écrit et pictural.

En dehors de cette limite institutionnelle, la ligne dissociant la production littéraire et les arts visuels renvoie également à une réalité conceptuelle: à une certaine représentation $\mathrm{du}$ monde dérivant directement de la hiérarchisation des sens et de l'opposition entre corps et esprit. Si la littérature a à voir avec le domaine des idées - le champ immatériel de la pensée -, les beaux-arts s'attachent pour leur part au monde des objets, à la réalité concrète. D'un côté, les inscriptions ne sont qu'un support matériel subalterne au développement de l'esprit; de l'autre, c'est la matière pigments mélangés à l'huile, marbre, bois, etc. - qui priment sur les idées. Les formes et les couleurs en appellent au toucher et à la vue bien moins qu'à l'intellect alors que l'écriture, elle, requiert un effort d'intellectualisation se passant de toute sensorialité.

Cette vision - idéal séparant la littérature et les arts plastiques en deux nations étrangères - a longtemps perduré jusqu'à ce que les recherches modernistes des XIX et $\mathrm{XX}^{\mathrm{e}}$ siècles viennent questionner l'arbitraire de cette frontière. 
Toutefois, malgré les recherches formelles des écrivains et les expériences poétiques des peintres, ceux-ci restent tributaires des systèmes établis de diffusion. Ainsi, si littérature et art - en tant que formes d'expression spécifiques - ont ouvert leur frontière l'une à l'autre, elles ne se sont jamais constituées en une nation unie. Le livre reste le voisin du tableau et de la sculpture, mais n'intègre jamais leur domaine en raison du lieu auquel il est attaché, les étagères des librairies ou des bibliothèques. De même, l'œuvre d'art ne peut se transposer dans un livre sans perdre ses caractéristiques d'œuvre d'art. Le musée, la galerie ou encore l'intérieur privé du collectionneur sont ses espaces de prédilection.

\section{Les deux régions du « brut » : les écrits et l'art}

Même si Jean Dubuffet a montré un intérêt pour les productions écrites dès ses premières prospections ${ }^{2}$ - des textes issus d'hôpitaux psychiatriques sont en effet rassemblés dès les années 1940 -, ce n'est véritablement qu'à partir des années 1960 qu'« un art brut dans l'écrire » (Thévoz, 1978, p. 12) est mis en lumière. À ce moment, la Compagnie de l'art brut, qui avait été dissoute en 1951, est reconstituée. Parmi les nouveaux objectifs de l'association, une place toute particulière est accordée aux formes écrites. Dans la « Notice» sur la nouvelle

\footnotetext{
2 À la suite de la dénomination de l'«art brut» en 1945, Dubuffet commence des prospections dans les hôpitaux psychiatriques et les campagnes (essentiellement en France et en Suisse). En 1948, il fonde la Compagnie de l'art brut afin de rationaliser ces collectes et d'éviter les dérives marchandes des œuvres rassemblées. Des expositions sont organisées dans les sous-sols de la Galerie Drouin à Paris, le Foyer de l'art brut.
} 
Compagnie, Dubuffet, faisant état des collections, précise notamment ceci :

Outre les œuvres à caractère proprement artistique, nos collections comprennent aussi quelques manuscrits ou écrits divers qui nous paraissent avec les normes de la littérature usuelle dans le même rapport que les ouvrages d'art brut avec ceux des arts culturels. (1967, p. 172)

Concernant les publications ${ }^{3}$ projetées par l'association, Dubuffet souligne, de plus, que le déchiffrement et la transcription de certains de ces textes sont prévus. Les fascicules à paraître intégreront ainsi non seulement «des reproductions d'œuvres les plus marquantes » de la collection mais aussi «des écrits [...] qui procèdent d'une position inventive analogue à celle de l'art brut » (p. 172).

Ainsi, par ces nouveaux objectifs, Jean Dubuffet intègre l'univers littéraire à sa pensée anti-culturelle et établit l'écriture aux côtés de la création plastique au sein du monde du «Brut». Toutefois, malgré son intention première - remettre en question ce qui structurait la culture occidentale, à savoir ses catégories artistiques, ses institutions et son goût, par le biais d'une nouvelle conception de l'art -, la manière de considérer les écrits au sein de l'«art brut» ne fait que réitérer une structure qu'il voulait déconstruire. En effet, la reconnaissance des formes écrites dans l'« art brut » reste tributaire de la frontière tracée culturellement entre littérature et art. Les textes «bruts» sont conçus dans un rapport d'analogie avec les œuvres d'art «brutes », et ces deux formes d'expression sont de nouveau isolées en deux univers parallèles.

\footnotetext{
${ }^{3}$ Une série de publications - les Cahiers de l'art brut - documentant l'œuvre et la vie de chaque auteur est mise en place (bien que planifiée en 1947, cette publication ne débute que dans les années 1960).
} 
Une dizaine d'années plus tard, la parution des Écrits bruts (Thévoz, 1979) ne fait qu'officialiser cette distinction. La définition qui en est donnée transpose littéralement le concept d'« art brut» dans le domaine littéraire. Alors qu'en 1949, Jean Dubuffet entendait par « art brut»

des ouvrages exécutés par des personnes indemnes de culture artistique, dans lesquels donc le mimétisme, contrairement à ce qui se passe chez les intellectuels, ait peu ou pas de part, de sorte que leurs auteurs y tirent tout (sujets, choix des matériaux mis en œuvre, moyens de transposition, rythmes, façons d'écritures, etc.) de leur propre fond et non pas des poncifs de l'art classique ou de l'art à la mode. (1967, p. 201202),

les « écrits bruts », eux, désigneraient

des textes produits par des personnes «ignorantes » ou réfractaires à la « culture des cultivés », insoucieuses des modèles du passé, indifférentes aux règles du bien-écrire (si ce n'est pour les transgresser), totalement étrangères en tout cas au milieu que nous associons aussitôt à l'idée de littérature, celui des écrivains, des éditeurs et des critiques. (Thévoz, 1979, p. 6)

Il est à noter que la publication des Écrits bruts correspond à un moment particulier de l'histoire de l'«art brut», à un moment d'institutionnalisation et de restructuration (de structuralisme aussi), pourrait-on dire, où la question des catégories semble importante. Au début des années 1970, en effet, Jean Dubuffet lègue les œuvres jusqu'alors rassemblées par la Compagnie de l'art brut à la ville de Lausanne. Cette donation mène, en 1976, à l'ouverture de la Collection de l'art brut, dont Michel Thévoz sera est le premier directeur. Bien que ce lieu d'exposition se veuille au départ un "anti-musée », les questions d'accrochage, de catégories ainsi que de discours sur les œuvres accrochées se posent tout comme pour une institution normale. Le concept 
d'« écrits bruts» se dessine donc à un moment où les textes qu'il désigne se voient accorder une nouvelle visibilité; le fait d'être exposés au sein d'un musée à côté d'œuvres d'art ayant comme suscité la nécessité d'en tracer les contours conceptuels.

Si l'apparition de la notion d' " écrits bruts » suit l'ancien modèle séparant les lettres et les arts, la place de l'écriture dans l'«art brut» s'avère toutefois plus complexe qu'une simple limite entre deux champs d'expression. La matérialité des textes - graphisme, rapport texte / image, néologismes, etc. - met en effet en question la frontière dressée entre œuvre picturale et œuvre littéraire et interroge par là notre manière d'appréhender traditionnellement un texte ou une œuvre d'art.

\section{Quand les mots deviennent formes et quand les mots assaillent les formes}

Le mot prend forme : il devient lui. Cette forme devient ce que le mot représente. Tout est mots. Tout ce qui est mot est plus ou moins forme? Tout ce qui est forme plus ou moins se pénètre, plus ou moins est pénétré, plus ou moins s'enveloppe, plus ou moins est enveloppé. («Palanc l'écrituriste »", dans Dubuffet, 1967, p. 230)

Comme le mentionne Michel Thévoz en introduction des Écrits bruts, la découverte d' « œuvres plastiques [...] étrangères dans une large mesure à notre culture» - œuvres qui furent qualifiées par Dubuffet d'« art brut » - a amené à « mettre à jour

\footnotetext{
4 Né à Vence en 1928, Francis Palanc était pâtissier. À l'âge de 19 ans, il commença à élaborer son système alphabétique, tout en réalisant parallèlement des tableaux faits à partir de coquilles d'œuf et d'autres matériaux périssables. Ce poème était inscrit sur une feuille collée au dos d'un de ses tableaux. La transcription donnée par Dubuffet conserve les fautes d'orthographe présentes dans le texte original.
} 
des processus de création et de subversion qui pouvaient emprunter presque indifféremment, pourrait-on dire, le moyen des formes figuratives et des mots de la langue » $(1979$, p. 10). Il précise d'ailleurs qu'un des traits caractéristiques des écrits bruts consiste justement «à ne jamais se détacher tout à fait d'une impulsion graphique qui peut aussi bien s'appliquer à une expression figurative», révélant par là "l'arbitraire des catégories telles que beaux-arts, littérature, musique, etc.» (p. 9-10).

Dans la plupart des "écrits bruts », le tracé du stylo peut en effet devenir forme ou lettre, modeler la surface de la feuille en un espace plastique ou être le simple support à un signifié. La ligne peut être lue et vue simultanément comme un signifiant ou comme le contour d'une figure plus ou moins abstraite. La frontière entre littérature et art se dissout alors pour laisser place au domaine plus large du geste graphique.

Les œuvres de Palanc dit l'écrituriste ${ }^{5}$ sont en ce sens révélatrices. Elles partent du désir de créer un alphabet qui puisse signifier les fondements universels, non par les lettres traditionnelles mais par une graphie guidée par des principes géométriques. La géométrie, selon Palanc, régit la vie mentale de chaque individu et c'est ce sens caché, ce lien profond qui unit l'homme au monde, qu'il cherche à mettre à jour à travers un code se situant à la limite de la lettre et de la figure. Personnifiant la géométrie, il écrivait: "Je suis géométrie visage universel. Mes lignes sont des mots - des murmures sans

\footnotetext{
5 Les écrits de Francis Palanc sont les rares écrits intégrés aux « écrits bruts » qui ne proviennent pas d'hôpitaux psychiatriques.
} 
bruit. Lentement, doucement, je vous pénétrerai. Faisant de votre forme - pureté de mes lignes. » (Dubuffet, p. 222-223)6

$\mathrm{Au}$ cœur de ses recherches plastiques et alphabétiques se trouvent les notions du masculin et du féminin. Le premier est signifié graphiquement par des tracés rectilignes et par le carré; le second, par des courbes et le cercle. Mais au-delà de cette analogie entre formes et principes universels, c'est l'idée de métamorphose et d'incarnation qui est à l'œuvre dans ses écritures. Le mot prend corps dans la forme et la forme donne vie à la chose signifiée. Ses exercices graphiques et géométriques visent en effet avant tout à trouver l'expression visuelle par laquelle le mot retrouvera toute sa vertu et sa consistance profonde. Ceux qui apparaissent le plus souvent dans ses tableaux - «Moi », « Toi », « Lui », «Vie», « Joie», «Vase» - s'insèrent les uns dans les autres et se métamorphosent pour mieux exprimer l'interrelation existant entre l'homme et la femme. Jean Dubuffet qualifie la création scripturale de Palanc d'«écriturisme» et d'«alchimie pronominale» (1967, p. 230), montrant par là que la limite entre domaine littéraire et artistique s'évapore face à un langage préoccupé avant tout de mettre à jour le sens et le flux de la vie.

Si la plasticité même des "écrits bruts" remet en question la frontière entre dessin et écriture, un autre trait de ces textes vient également perturber cette limite: la

\footnotetext{
6 En plus des inscriptions présentes dans ses tableaux, Palanc rédigea de nombreuses notes - entre simples notations, aide-mémoire et poèmes - pour améliorer son système alphabétique. Le texte dans lequel il donne la parole à la Géométrie - et duquel cette citation est tirée - correspond à ce pan de sa création.
} 
juxtaposition des textes et des images dans un rapport qui s'avère plus complexe que celui d'une simple illustration.

Dans les écrits de Joseph Heuer7, par exemple, la mise en page des dessins et des écritures brouille le dialogue traditionnel qui s'établit entre le langage pictural et le texte dans un livre imagé. Le dessin est une sorte de pictogramme qui constitue un signe à part entière autour duquel est agencé le texte. Les mots ne suivent pas la linéarité et la verticalité conventionnelles de l'écriture occidentale, mais exploitent la spatialité de la feuille dans un rapport analogique avec ce qu'elle signifie. Joseph Heuer, qui était sergent dans l'infanterie avant d'être interné, conçoit en effet l'espace de la feuille comme un territoire qu'il investit par les mots et les images. Ses «tableaux de graphismes », comme les nomme Jean Dubuffet,

ouvragés avec grand soin d'une fine plume, en plusieurs couleurs, et avec beaucoup d'inscriptions portées à l'envers fonctionnent à la fois [...] dans le sens de libellés et dans celui de topographies : ils sont en même temps des actes et des cartes, quand même ils n'assument pas encore après cela des figurations à la signification symbolique. (1967, p. 288)

Les frontières, par exemple, sont marquées par des baïonnettes - petits signes anguleux qui délimitent les territoires tout en manifestant sur le mode symbolique « la garde militaire et la présence vigilante en tous lieux du sergent lui-même » (p. 289). Concernant les écritures même de ses dessins, Joseph Heuer

\footnotetext{
7 Joseph Heuer (1827-1914) est interné en 1860 à Genève jusqu'à la fin de sa vie. Ce n'est que tardivement, vers 1907, et après avoir changé d'asile, qu'il semble avoir commencé à écrire et à dessiner. C'est grâce au Professeur Ladame que ses dessins et écrits ont été préservés. On sait peu de choses de Joseph Heuer, simplement qu'il a été ébéniste, relieur, régleur de registres avant d'être sergent dans l'infanterie. C'est cette dernière profession qui ressort le plus dans son œuvre.
} 
greffe à ses mots de nombreuses lettres muettes, accentuant par là la dimension graphique de l'écriture et l'aspect visuel des mots. «Territoire » devient «TERRHITHOIRE » et les pays qui apparaissent sur certaines de ses cartographies - l'Italie, la Russie ou encore l'Amérique - prennent les formes respectives de «Hithalhie», «Rhusscies» et «Hammerhic». Si ce maniérisme orthographique semble inutile sur le plan du sens et rend difficile, au premier abord, la compréhension du texte, il joue un rôle sur le plan visuel. D'autre part, il révèle une troisième dimension du langage, son oralité. Une fois lues à haute voix, en effet, les parties silencieuses des mots se taisent et le sens caché derrière des inscriptions visuellement incompréhensibles est dévoilé.

La plasticité même de l'écriture dans les «écrits bruts » tout comme la présence simultanée d'inscriptions et d'images ou encore les néologismes purement phonétiques engagent un mode de lecture qui ne met pas seulement en jeu le processus d'intellection - comme dans la lecture d'un livre imprimé ou d'une lettre traditionnelle - mais qui implique aussi de prendre en considération l'aspect visuel et sonore du texte. Ces dimensions viennent perturber la limite séparant les modes de diffusion de l'art et de la littérature, à savoir respectivement l'exposition et la publication. Les textes relevant des "écrits bruts » sont à la fois à voir, à parler - c'est-à-dire à déchiffrer à haute voix - et à entendre - c'est-à-dire à lire comme un message. Leur transcription typographique - format exigé pour leur publication - ou bien leur mise sous verre - mode de présentation muséal - limitent obligatoirement un de ces aspects. La typographie omet la richesse formelle de la graphie - sa disposition sur la feuille, le style même du tracé, etc. - pour se concentrer sur les particularités linguistiques de l'écriture, 
alors que leur accrochage rend difficile la lecture des textes pour se focaliser sur leur dimension esthétique - les pages, dans le cas de livres, ne peuvent être feuilletées et la feuille ne peut être tournée pour suivre des inscriptions courant dans plusieurs sens.

Ceci n'est pas pour pointer du doigt la déficience de ces modes de diffusion : leur présence est nécessaire et a permis de faire reconnaître des écrits qui, sans eux, seraient restés dans l'ombre. Plutôt, cette analyse vise à montrer que les textes relevant des "écrits bruts» se situent au seuil de deux catégories. Par cette position même, ils perturbent la stabilité de la frontière entre littérature et art. Ils la rendent perméable, la déplacent, la traversent, apportant tour à tour des éléments de l'art dans le monde de la littérature et vice-versa, ceux de la littérature dans l'univers de l'art. Ils sont une sorte d'interface où dialoguent littérature et art. Toutefois, ils sont également un troisième monde dont les occupants semblent condamnés à osciller entre deux pays auxquels ils n'appartiennent pas entièrement.

\section{La stratégie de la frontière dans le discours}

sur les «écrits bruts»

Comme il est mentionné précédemment, la dimension visuelle et orale des "écrits bruts » tend à les situer en marge de la littérature. Toutefois, cette position - si elle correspond à une réalité matérielle - est renforcée par le discours avant-gardiste qui leur est associé. En effet, la notion de marginalité constitue un élément stratégique dans la définition des « écrits bruts » et contribue à en faire un outil critique de la littérature 
traditionnelle. Et Michel Thévoz de dire : « La notion d'écrit brut se définira essentiellement par opposition à la littérature telle qu'on l'entend ordinairement. » (1978, p. 11)

\section{Pour un contour historique des " écrits bruts »}

Pour mieux comprendre le discours associé aux " écrits bruts", il y a lieu de revenir sur le contexte qui lui a donné le jour. Le moment de reconnaissance des «écrits bruts" - à savoir les années 1960 et 1970 - correspond à un contexte littéraire et intellectuel particulier. Le langage est alors au centre des débats : Richard Rorty parlait à ce propos de "linguistic turn » (1967). La scène culturelle française est marquée par une attirance certaine des sciences humaines par la linguistique et la sémiotique. Sous l'influence du structuralisme, le langage est posé comme un système interrelationnel mettant en jeu les mots, les signes, les individus et le pouvoir. Son arbitraire tout comme sa relativité et sa normativité sont portés sur le devant de la scène. L'idée d'un langage individuel, ingénu et naturel est abandonnée au profit de la vision d'un langage autoritaire et aliénant. À cette vision pessimiste, s'ajoute la désillusion d'une possibilité de nommer la réalité. Les mots ne sont pas les choses et échouent dans leur tentative de cerner le monde matériel. D'autre part, dans le domaine de la communication, le développement de nouveaux médias contribue à faire du langage un outil purement objectif, strictement voué à véhiculer du sens et à reléguer sa dimension poétique dans les limbes de l'oubli. Face à cette situation du langage, nombre d'écrivains s'interrogent sur le sens à donner à la littérature. Dans la continuité de la pensée barthésienne selon laquelle «il n’y a rien en dehors du langage », ils se confrontent à l'impossibilité 
pour le langage de signifier l'humain et la réalité. La forme des mots, et non ce qu'ils portent - leur sens - est alors explorée pour tenter de dire ce qui ne peut être dit. Dans le domaine des arts visuels, une même attention est portée au langage. Les néoavant-gardes, telles que le lettrisme ou encore Fluxus, accordent une place nouvelle à la poésie. Perpétuant l'assaut des catégories, elles intègrent les expériences littéraires à leur processus de création artistique. Elles tentent de relier l'art et la vie en abaissant les frontières entre poésie, art visuel et expérience du quotidien, au sein d'œuvres dans lesquelles les mots, les formes et les matériaux cohabitent.

\section{Contre l'objectivité du langage, pour une langue individuelle et poétique}

L'intérêt porté aux «écrits bruts » - textes majoritairement réalisés en contexte asilaire et mettant en jeu le langage de la folie - ainsi que la conceptualisation de ce terme doivent être restitués sur cette toile de fond. Dans les premiers ouvrages consacrés à ce concept - Le Langage de la rupture, 1978 et Les Écrits bruts, 1979 -, Michel Thévoz fait directement référence aux théories linguistiques et aux modèles de communication pour mieux les critiquer. Partant du système saussurien, il révèle comment ces éléments structurels sont l'objet d'une recomposition dans les "écrits bruts » et comment ces derniers se jouent du talon d'Achille de la linguistique, c'est-à-dire de l'arbitraire du signe. Dans les «écrits bruts », cette faille est en effet exploitée sous toutes ses facettes: des modifications orthographiques du signifiant (comme l'ajout de lettres inutiles, la suppression des lettres muettes ou encore le renchérissement des préfixes ou suffixes) en passant par 
l'exploration des variantes phonétiques ou encore des structures grammaticales (le verbe devenant un nom; le nom, un adjectif ou un adverbe, etc.).

Dans les écrits de Samuel Daiber ${ }^{8}$, par exemple, le suffixe «-ment» (propre aux adverbes) devient le moyen de rallonger des mots qui lui semblent trop courts. La négation «pas»se transforme en «pasement », «point » en «pointement», « ni » en «niment», ou encore « jamais» en « jamaisement». Sur le même modèle, Samuel Daiber invente de nouvelles expressions comme «automobilement» pour signifier "au moyen d'une automobile ». À partir de noms, il crée également de nouveaux verbes. Par le simple ajout de la terminaison «-er», il condense des expressions et rend actifs des objets au départ passifs. «Pistolader» («tuer au pistolet») redonne ainsi le pouvoir d'action au pistolet, "voixer » redonne la parole à la « voix», ou encore «périler» dit dans un raccourci l'ensemble de mots «mettre en péril »

Dans l'analyse des écrits dits «bruts ", l'accent est placé sur la logique subversive du processus de réécriture. Le détournement de l'écriture - selon le titre de l'ouvrage de Michel Thévoz (1989) - qui mène aux «écrits bruts » récupère les règles linguistiques et se joue de leur caractère conventionnel pour aboutir à un langage individuel déroutant. Les dérives graphiques font glisser le rapport établi entre le signifiant et le

8 Samuel Daiber, né en 1901, est hospitalisé de manière permanente à l'âge de 47 ans (il meurt en 1983). À partir de ce moment, il se met à écrire des textes qui « ont la forme de longues missives adressées au médecin directeur de l'établissement, à des parents ou à des destinataires imaginaires. Ce sont pour la plupart des feuilles de papier à lettre lignée, de $30 \times 21 \mathrm{~cm}$, que Samuel D. couvre intégralement d'une écriture soignée et régulière en biffant parfois de longs passages [...]. » (Thévoz, 1979, p. 61-86) Les textes de cet auteur ont été l'objet d'une étude approfondie (Capt, 2012). 
signifié. L'indépendance du signifiant s'affirme : le mot peut être écrit indifféremment sous telle ou telle orthographe sans pour autant affecter le sens du signifié. Réciproquement, un même signifiant peut renvoyer à plusieurs signifiés; sa disposition sur la page, sa plasticité et sa sonorité offrant plusieurs possibilités d'interprétation visuelle et orale. Dans les écrits d'Annette Libotte $^{9}$, par exemple, les règles phonétiques sont mises à jour dans l'orthographe même des mots : «l'eswi min » correspond à «l'essuie-mains», «le trois mai » devient «le trwa m est»mais on pourrait tout aussi bien lire «le tramway»-, "nos ailes » sont décomposées en «no z'estl» - mais de nouveau, d'autres mots peuvent s'envoler de cette orthographe selon l'imagination du lecteur, tels que « zèle » ou « zeste »10.

Un autre aspect qui est mis en avant dans l'analyse des " écrits bruts » est leur dimension anticommunicative: nous souhaitons préciser ici que cet aspect est lié aux particularités stylistiques des «écrits bruts » bien plus qu'à l'intentionnalité de leurs auteurs. La réformation et reformation des règles linguistiques observables dans la majorité de ces textes vont, en effet, à l'encontre du modèle de communication qui conçoit la langue comme un lien univoque reliant un émetteur et un récepteur.

Ainsi, le glissement de l'arbitraire du signe vers la libération de ses ressources sémantiques et graphiques

\footnotetext{
9 Annette Libotte est née à Bruxelles en 1890. Elle est internée à plusieurs reprises en Belgique à partir de 1934. Les textes conservés à la Collection de l'art brut et reproduits dans l'anthologie Écrits bruts ont été écrits en 1941 et 1942. Ils consistent en « 350 pages réparties dans deux petits carnets, genre bloc-notes, de 13 x $8 \mathrm{~cm}$ » (Thévoz, 1979, p. 41-54).

10 Nous nous sommes permis d'ajouter à la transcription classique (telle que proposée dans les Écrits bruts) d'autres évocations, celles mêmes que ces graphies ont suscitées en nous, indépendamment du sens du texte.
} 
contribue à faire des "écrits bruts" un cheval de Troie permettant d'ébranler la conception rationnelle du langage. Ceux-ci sont une entrée par laquelle le discours anticulturel de Jean Dubuffet et de Michel Thévoz vient critiquer sa normativité et sa fonction coercitive au sein de la société. Comme des feux de Bengale lancés dans un monde en perte d'altérité, les « écrits bruts » sont récupérés pour éclairer la possibilité d'une expression plus individuelle et poétisée résistant aux conventions culturelles et à l'uniformité sociale.

Prenant comme support les textes de Samuel Daiber, Michel Thévoz disait notamment à ce propos :

Contrairement au commun des individus pour qui l'homéostasie (c'est-à-dire la contrainte de stabilité) du langage représente une garantie de communicabilité, Samuel, rejeté de toute manière du circuit de la communication, la ressent comme une gêne. Pour réagir contre la normalisation du langage, contre le refroidissement et l'oubli des métaphores, contre l'effacement de l'étymologie, il entreprend de maîtriser individuellement le processus d'évolution. (1978, p. 164-165)

Enfin, les spécificités linguistiques des «écrits bruts» sont mises en parallèle avec les recherches des poètes de l'époque. Les déformations orthographiques, la transposition de l'oralité du langage dans l'écriture ou encore les jeux formels de la graphie sont en effet au centre des préoccupations d'aprèsguerre. Jean Dubuffet, aux côtés de Raymond Queneau, insistait notamment sur le fait que la généralisation de la typographie en Occident « avait fait oublier toutes les ressources visuelles des graphies» et «toutes les ressources du parler» (Dubuffet, 1967, p. 293) et que l'écriture se devait de retrouver son expression plastique et verbale avec ses « sonorités, inflexions, mimiques» (p. 293). De même, Raymond Queneau, s'amusant 
des règles de la langue française, proposait «non de corriger l'orthographe de l'ancien français [...] mais de choisir quelle orthographe donner au nouveau français », en précisant que « la plus phonétique semblerait s'imposer » (Queneau, 1965, p. 22). Dans un autre registre, Francis Ponge, face aux débats sur le silence de la poésie à la suite des atrocités de la Seconde Guerre mondiale, faisait du «jargon» l'espoir de ce genre11. Ainsi, ce n'est pas par hasard si les poèmes de Queneau, de Ponge ou encore les Pièces littéraires de Dubuffet sont intégrés, de manière comparative, à l'analyse des «écrits bruts ». Ce dialogue permet de renforcer le potentiel expressif et poétique des " écrits bruts » et d'éclairer le fait que la langue est bien plus qu'un système régi par des conventions ou un instrument de communication facilement maîtrisable. Face à un langage pris dans l'histoire et les normes littéraires, les «écrits bruts » sont présentés comme une porte libératrice : celle de la folie et de l'altérité.

Le discours sur les "écrits bruts » participe ainsi de la défense d'un langage autre et d'une écriture plurielle qui répondent à l'exhortation des poètes, à savoir explorer " cette vertu stupéfiante des mots lorsqu'on les dispense de la transmission des idées et qu'on réveille ce qu'on pourrait appeler leur processus primaire » (Thévoz citant Paul Valéry, 1978, p. 153). Il participe également d'une nostalgie plus générale, d'un fantasme collectif cherchant à retrouver un état utopique du langage, un langage pré-institutionnel où écriture, parole et gestualité exprimaient l'homme dans son essence

11 Répondant à la constatation de Theodor Adorno, «Écrire un poème après Auschwitz est barbare », Francis Ponge écrivait : «L'espoir est donc dans une poésie par laquelle le monde envahisse à ce point l'esprit de l'homme qu'il en perde à peu près la parole, puis réinvente un jargon. » (1961, p. 195-199) 
même, une langue idéale où les mots approchaient de si près les choses qu'ils en captaient le sens profond, un langage individuel libéré de toute contrainte sociale.

\section{Les " écrits bruts ", " un pôle voguant à contre-courant » 12?}

Le discours sur les "écrits bruts » tend à reconstituer ce lieu fantasmé du langage. Dans cette construction, le tracé de la frontière est central. Toutefois, celui-ci n'est pas neutre. Comme en situation de guerre, il établit un rapport de force entre le lieu des « écrits bruts » - territoire de la folie - et son parti ennemi celui de la «culture». Les normes sociales et l'usage conventionnel du langage sont présentés comme l'empire hégémonique à ébranler; les "écrits bruts» et leur auteur, comme le char d'assaut. Le pouvoir des " écrits bruts » est vu, en effet, dans leur capacité à «s'en prendre à la base la plus résistante du conditionnement culturel, sa racine, c'est-à-dire aux mots et à leur agencement usuel » (Thévoz, 1978, p. 8).

Cette attaque est modélisée selon différentes topographies, de la vision du traître qui attaque de l'intérieur à celle du révolté qui a rompu avec le système établi ou encore de l'étranger venant de l'extérieur. Toutefois, quelle que soit la spatialisation choisie, le discours sur les " écrits bruts » en revient toujours à l'opposition entre le centre et la périphérie: le centre correspondant à ce qui est reconnu par tous, au contrat social, à la norme; la marge, à ce qui est autre, à la différence, à la folie. À ce propos, Michel Foucault disait d'ailleurs :

Ainsi ont-ils tous les deux le fou et le poète, au bord extérieur de notre culture et au plus proche de ses partages essentiels, cette situation «à

12 Cette expression fut employée par Jean Dubuffet pour redéfinir l'art brut en 1959 (1967, p. 515). 
la limite » - posture marginale et silhouette profondément archaïque où leur parole trouve sans cesse leur pouvoir d'étrangeté et la ressource de leur contestation. (1966, p. 64)

S'agissant de la première topographie - celle du traître -, l'idée maîtresse est que les auteurs d' « écrits bruts » connaissent et partagent la langue des hommes de culture, mais qu'ils en usent de manière pernicieuse, la détournant de son usage habituel pour la diriger vers un état limite auquel peu ont accès, aux confins de l'hermétisme sémantique et de l'illisibilité :

Les auteurs d'écrits bruts ne parlent pas une autre langue que les écrivains professionnels [...]. Il faudrait plutôt les considérer comme des intrus dans leur propre langue, comme des voleurs, qui procèdent par rapts systématiques trahissant le sens des mots et perturbant les convenances de la syntaxe. (Thévoz, 1978, p. 12-13)

Dans le second type de cartographie, les auteurs d'«écrits bruts » sont positionnés en dehors du système culturel, dans des îlots de désaliénation sociale que sont les asiles. Leur "position d'esprit rebelle à toute norme et à toute valeur collective» les a marginalisés. "Réfractaires au dressage éducatif et au conditionnement culturel», ils se sont «retranchés» de la collectivité (Thévoz, 1990, p.34). En choisissant la folie comme mode de pensée, ils ont toutefois gagné une victoire: celle de ne plus devoir répondre aux exigences de la communication et de parler une langue qui leur est propre. Sortes d'«anarchistes » et de "terroristes», selon les mots de Michel Thévoz, ils «détournent les mots pour produire des textes socialement inassimilables. » (1978, p. 180) Leur folie est ainsi assimilée à une voie de libération qui part d'un état de révolte contre le poids de l'impérialisme culturel et mène vers les marges de la raison. 
De ce modèle qui présente les auteurs d'« écrits bruts » comme des "refuseurs et autistes » (Thévoz, 1990, p. 34) isolés dans l'asocialité, dérive le troisième, à savoir l'idée qu'ils sont aussi des étrangers qui peuvent attaquer de l'extérieur. Habitants de l'ombre, ce lieu méconnu qu'est la folie, ils sortent parfois de cette nuit en se manifestant sur la page blanche. Toutefois, quand ils se mettent à écrire, ce n'est pas par "référence familière à des auteurs aînés », mais poussés par « un sentiment de non appartenance qui se résout par une agression inventive contre le langage.»(Thévoz, 1978, p.12) Les textes qu'ils produisent - sorte de langage de la rupture abattent la frontière codifiée de la littérature et infiltrent la folie dans son champ en venant perturber son ordre.

Dans chacune de ces représentations spatiales, l'intentionnalité des auteurs d' "écrits bruts» est modelée et redirigée selon la visée critique du concept d'«écrits bruts». Les auteurs sont positionnés comme les sujets de la guerre menée contre la littérature, la culture et les règles sociales, ce qui est en grande partie spéculation interprétative. De la même manière, les textes relevant des « écrits bruts »- objets inertes au départ - tendent à recevoir un pouvoir d'action anticulturel. Ce pouvoir n'est pas intrinsèque aux écrits mais découle de la construction discursive qui les entoure. Cette dernière, nourrie de sciences humaines et faisant écho aux enjeux du monde littéraire contemporain, leur donne les rames et les armes pour qu'ils voguent à contre-courant.

Nous souhaitons, dans une troisième partie, considérer ces écrits sous une lumière différente. Mettant de côté le rapport de force établi dans le discours de Jean Dubuffet et de Michel Thévoz entre les "écrits bruts » et la littérature, nous 
voudrions concevoir ces productions comme une interface mettant en jeu du sens, au-delà de toute question de catégorie.

\section{Traverser la frontière du champ de la folie}

\section{Au sein des murs de l'asile}

Comme il est mentionné en introduction, les textes dont il est question dans les "écrits bruts " sont issus majoritairement d'hôpitaux psychiatriques. Datés pour la plupart d'avant 1950, ils ont été réalisés dans un contexte particulier. En effet, avant la seconde moitié du XXe siècle, l'internement signifiait le plus souvent la réclusion à vie, notamment pour les patients "schizophrènes » qui étaient placés dans des pavillons spéciaux. Les relations humaines au sein de l'asile étaient limitées et régies par le code de déontologie de l'époque, à savoir éviter tout rapport de réciprocité entre le personnel médical et les malades pour contrôler les conséquences du transfert freudien. D'autre part, le discours du malade n'était pris en compte que pour sa valeur psychopathologique, et sa portée signifiante désamorcée du fait de sa folie. Les écrits qu'il produisait étaient préservés comme documents psychiatriques. Le langage qu'ils mettaient en scène était lu comme la manifestation de ses délires et la preuve de son incapacité à dialoguer normalement. Le patient était ainsi déresponsabilisé de ses paroles et isolé de l'autre côté de la « ligne de ségrégation entre la raison et la déraison». Cette situation «bloquait d'emblée le mouvement de réciprocité que le langage appelle par principe » (Thévoz, 1978, p. 44). De plus, les contacts avec l'extérieur étaient extrêmement limités : les visites étaient rares 
et les sorties quasi inexistantes; quant au courrier, il passait rarement la frontière de l'asile ${ }^{13}$.

Langage de la rupture, les " écrits bruts » le sont. Parce qu'ils rompent avec les normes linguistiques certes, mais aussi parce qu'ils tentent d'abattre les murs du silence qui entoure leur auteur. Ces textes nous donnent à voir une langue qui essaie sans fin de communiquer mais n'y parvient pas faute d'écoute et de destinataires. Si anticommunication il y a, ce n'est donc pas par refus de communiquer mais plutôt en raison de la barrière qui s'impose à ces messages. Au-delà de la question du sens - c'est-à-dire l'aspect inintelligible de certains de ces écrits -, il y a donc lieu de revenir sur l'acte d'écrire en soi, qui consiste déjà en une tentative de faire sens. Toute inscription peut en effet être lue comme la volonté de laisser une trace, de marquer un support d'une présence, de faire mention de quelque chose ou d'ouvrir sur une réalité autre : «Tu ne traces rien, ce que tu vie seulement te trace dans la mesure ou ce que tu vie est inconnu de tous même de toi. » (Palanc l'Écrituriste, dans Thévoz, 1978, p. 81)

\section{Une écriture auto-suffisante}

« The outsiders thus lives to be enclosed in the radiant space of his own creativity. It is a self-sufficient domain. » (Cardinal, 1979, p. 29)

Si l'internement impose au patient une coupure avec la société, il a également pour conséquence de reléguer aux frontières de l'asile son identité passée. Le patronyme qui a pour rôle d'inscrire un individu dans une lignée généalogique et de marquer son appartenance à une communauté perd sa valeur

${ }^{13}$ Sur le contexte asilaire, voir Quétel et Postel (2012). 
identitaire pour ne devenir qu'une simple dénomination (il est d'ailleurs le plus souvent éludé par le secret médical dans le cadre public). Quant à la profession, élément important de reconnaissance sociale, elle n'est plus permise au sein de l'hôpital. Face à cet anonymat, certains prennent le crayon et tentent désespérément de récupérer ce qui leur a échappé. Josome Émile Hodinos (1853-1905) ${ }^{14}$, par exemple, interné en 1876 et auteur de nombreux projets de médaille, retrouve dans le dessin un moyen de préserver ce qu'il était. Transposant son métier de graveur sur un autre plan - des papiers destinés à la confection de biscuits lui servent de support, et le crayon et l'encre font office de stylet -, il fait de sa propre effigie l'objet de ses pseudo-gravures. Les inscriptions qui courent au recto et au verso de ses dessins viennent décrire le sujet de la médaille, à savoir l'auteur lui-même. Les mots se suivent les uns à côté des autres, alignant les éléments le caractérisant, tels que des données sur sa famille, des informations concernant son activité professionnelle ou encore des détails sur ses faits et gestes au sein de l'hôpital. Dans un de ses dessins ${ }^{15}$ notamment, le premier paragraphe du texte nous présente ses origines et sa formation de graveur :

Émile Hodinos Josome. Connétable. Volontaire d'un an. Communard. Sabre de 1848. Émile Jean Charles Hodinos, son Père. Généralissime de armées de la République. Boulanger. Patron - trois boutique, laissa en mourant à son fils E. Josome Hodinos la somme de 25000 francs venu ou provenant de la vente de fonds de commerce Boulangerie. [...] Sortie de Pension à l'âge de 16 ans, où il avait été pensionnaire pendant 4 ans, une

\footnotetext{
14 De son vrai nom Joseph Ernest Ménétrier, il adopta celui de Émile Josome Hodinos une fois interné à l'asile de Ville-Évrard (Paris).

15 Le dessin dont ce texte est issu est préservé à la Collection de l'art brut; il est écrit à la plume sur une feuille de 32,5 x $21 \mathrm{~cm}$ et daté entre 1876 et 1896. Les fautes d'orthographe du texte original ont été conservées.
} 
année Externe - Il entra chez le Patron Tasset, Graveur en Médailles.

Il nous donne également des détails sur sa personne physique à la manière d'une description anatomique :

Émile Josome Hodinos. Est d'une taille moyenne. La grandeur de sa tête égale la distance comprise sous le menton au dessous des Pectoraux, des Pectoraux au nombril, du nombril à la ligne $\mathrm{du}$ ventre, ses épaules mesure deux têtes de longueur, sa Poitrine bombé on y lit les côtes, le bout du Sertorum - les clavicules, les humérus, le bassin, les têtes de fémurs, les Cubitus et radius.

L'écriture n'en finit pas de le répertorier dans une dynamique tautologique, ce qu'il est dépendant de ce qu'il a été, de ce qu'il a fait et de ce qu'il fait au moment présent. La description de son identité et de son physique semble ne pas suffire à la captation de sa personne. Il en vient à décrire le processus de création luimême, l'écriture ayant comme besoin de se raconter elle-même pour dire l'identité de celui qui écrit. Dans le dessin décrit précédemment, le recensement de ses gestes d'artiste et des créations qu'il a réalisées ou qu'il est en train de réaliser au sein de l'asile prolongent les informations biographiques à la manière d'un chapelet sans fin :

Enfermé à la Ville Evrard le 15 Décembre 1876. [...] Écrit sa Biographie. [...] Décrit son enfermement à la Ville Evrard. [...] Dans mes 10 volumes de médailles réunis pour savoir ce que c'est que la médaille, j'y ai passé onze année. J'ai divisé des feuilles de papiers biscuits en deux dans le sens de la hauteur puis en deux dans le sens de la largeur ce qui ma donné 4 petites feuilles pour une. J'en ai ensuite coupé 200 autres [...]. (Thévoz, 1979, p. 106-113)

$\mathrm{Si}$, dans les écrits de Hodinos, la quête de sens se fait par l'inventaire d'une identité en voie de disparition, dans d'autres écrits, elle se manifeste par des requêtes auprès d'autorités 
réelles ou fictives, mais toujours muettes. Les lettres de Samuel Daiber - en dehors des néologismes qu'elles présentent et dont nous avons parlé plus haut - s'avèrent de véritables protestations contre l'enfermement : "Je Demande Comande à sortir de ce Peureux, à le quitter; je ne veux pas que l'on le perpétue. Je m'oppose à ce que l'on me conduise, que l'on m'enfermeture de nouveau, en, dans un Hospice; je ne veux pas. on est pas chez soit. [...] Je veux mes droits civiques come chacun. Je ne veux pas que l'on me traite en désuette. Je suis normal. » (Thévoz, 1979, p. 63)

De la même manière, Gaspard Corpateaux (1838-1917) ${ }^{16}$, qui adopte le discours formel des lettres officielles - il était avocat avant d'être interné -, rédige des missives afin d'être libéré. Tout comme chez Émile Josome Hodinos, la question de l'identité est centrale; notamment dans sa signature, qui est en quelque sorte le lieu de son incarnation. Gaspard Corpataux marque en effet toutes ses lettres de son nom, de ses initiales complètes, de son statut de citoyen et de son ancien métier d'avocat. Il fait également état du temps écoulé depuis son entrée à l'asile en inscrivant la date de son internement - 1880 - et la date à laquelle il écrit à la fin de chaque lettre. Alternant l'encre rouge et noire, et cadrant ses inscriptions au centre de la page, il adresse ses propos le plus souvent sous cette forme :

Salut. Médecin-Directeur. C'est "assez" dénigrer Injurier mépriser: D’office au grand devoir En bon Sauveur de droit Finissant dans l'endroit. Bien guérir le malade Et me sortir guéri Par vois soins corps-esprit Ordonnez mon départ Avec le

16 Avocat originaire de la ville de Fribourg, Gaspard Corpataux rédigea ses lettres entre 67 et 80 ans alors qu'il était interné à l'hospice de Marsens. Il les adressait majoritairement au personnel médical et aux membres du Conseil d'État de Fribourg (Adam et Capt, 2008, p. 72-79). 
nécessaire A teneur de la loi Marsens 1880-1906. G.M.B. Corpataux citoyen-avocat ${ }^{17}$.

Ainsi, dans un lieu où le silence est imposé aux mots du malade, ces textes révèlent la volonté de parler malgré tout. Sur le mode épistolaire ou encyclopédique, les auteurs de ces écrits tentent d'ouvrir un espace de communication. Faute de considération et de réponse, ils réitèrent sans cesse cette percée vers l'autre. Cette écriture "auto-suffisante » s'alimente à la source de leur passé et essaye de faire survivre le sens par le ré-ancrage - réencrage - de leur individualité.

\section{Les murs ont des oreilles}

Les auteurs d'écrits bruts s'expriment d'abord pour euxmêmes, et, s'ils s'adressent à des lecteurs, il ne s'agit aucunement de "public cultivé » familier des librairies, mais d'interlocuteurs imaginaires; s'ils visent la publication, c'est sur un mode utopique qui exclut d'emblée l'édition régulière de leurs textes. (Thévoz, 1979, p. 6)

Si la porte de l'asile est fermée sur l'extériorité, un voyage est toujours permis par l'imaginaire. Cet exil intérieur (Jaccard, 1975) amène à la création de véritables utopies. L'écriture « autosuffisante » s'alimente alors à la source des délires :

L'œuvre est donc envisagée par son auteur comme un support hallucinatoire. [...] Le processus créatif se déclenche aussi imprévisiblement qu'un épisode psychotique, en s'articulant

17 Ce texte correspond à une lettre conservée à la Collection de l'art brut: Salut Médecin-Directeur. Vérité, 1906, cab 10881. Dans la transcription que nous en donnons, les majuscules sont le fait de l'auteur et elles correspondent le plus souvent au début d'une nouvelle ligne. La plupart des mots sont soulignés en rouge, notamment le mot "sortir», qui est souligné plusieurs fois et positionné au centre de la page. 
selon sa logique propre, comme une langue inventée. (Thévoz, 1990, p. 35)

La narration d'une réalité parallèle devient un moyen de trouer les murs du silence et d'élargir le champ étroit de l'asile. Les murs mêmes de l'hôpital se transforment en support de ces histoires. Fernando Oreste Nanetti (1927-1994)18, interné à l'hôpital de Volterra en Italie, a ainsi pris les parois extérieures de l'édifice pour se raconter. Pendant neuf ans, lors des sorties régulières dans la cour de l'asile, il grava un gigantesque « livre de pierre » - libro graffito - avec la pointe métallique de la boucle de son gilet. Sur les soixante-dix mètres de façade de la cour intérieure de l'hôpital, il recréa son identité et son histoire. "Astronaute», "ingénieur des mines du système mentale», « colonel astrale », il raconta ses relations avec l'au-delà et ses voyages imaginaires. Sorte de missionnaire et médiateur en contact avec des forces cosmiques - il se prénommait « NOF4 » ou « Nanof » dans son histoire -, il rapporta, au sein des pages qu'il délimitait sur la surface du mur, les informations qu'il recevait par télépathie. Orphelin de père, il se reconstitua également une famille à travers l'écriture ${ }^{19}$.

Dans l'œuvre de Nanetti, le support et le format de sa création littéraire font sens en eux-mêmes, rajoutant au contenu des mots une dimension supplémentaire. Le développement spatial propre à l'écriture s'applique dans l'espace réel, dépassant ce qui apparaît au départ comme une limite matérielle, le mur de l'asile. Comme un déroulé cinématographique, elle permet à son auteur de «se faire son film » et de le projeter aux yeux de tous (ses inscriptions ne

18 Né à Rome, Fernando Oreste Nanetti entre à l'hôpital de Volterra en 1958. Il réalisa son livre de 1959 à 1972 (il sort de l'hôpital en 1973).

19 Pour plus d'information, voir Nanetti, 2011. 
dépassent pas la hauteur d'un homme). Elle opère en quelque sorte à cœur ouvert, éprouvant le mutisme des murs en les taillant, pour mettre à jour un imaginaire délirant. De plus, les inscriptions marquent le mur d'une empreinte indélébile: la pensée de son auteur y reste gravée durablement. Épreuve unique, difficilement destructible et indissociable de son lieu de création, ce livre se donne à voir, attendant un passant pour être lu. Anticommunicatif et autistique, il peut paraître du fait de l'utopie singulière qu'il met en scène. Mais au-delà de ce langage à sens unique, sa forme même invite à la lecture et s'ouvre à la postérité. Il s'agit ici à nouveau d'une question de frontière, non plus celle qui exclut mais celle qui est à traverser de la part de spectateur: faire le pas d'entendre ce qui se dit derrière les murs de la folie, de comprendre ce qui s'y cache et d'aller audelà du sens commun. Ce seuil, c'est un infirmier qui l'a franchi du vivant de Nannetti, Aldo Trafeli. Il a permis la préservation de cette œuvre quand celle-ci était menacée. À la fin des années 1970, les autorités italiennes lancèrent en effet le projet de fermer toutes les institutions psychiatriques. Aldo Trafeli se chargea alors de faire photographier les inscriptions de Nanetti. Cette initiative suscita, plus tardivement, l'intérêt d'autres personnes. Les murs furent reproduits par moulage pour être exposés et permettre à l'histoire de Nanetti de retrouver la parole et l'écoute qu'elle cherchait. Comme le disait Lacan, «il n'y pas de parole sans réponse, même si elle ne rencontre que le silence, pourvu qu'elle ait un auditeur » (1966, p. 247). 


\section{Bibliographie}

ADAM, Jean-Michel et Vincent CAPT. (2008), « Les lettres asilaires de Gaspard Corpataux. Poèmes de l'enfermement », dans Art brut fribourgeois, Lausanne / Fribourg, Collection de l'art brut / Éd. La Sarine.

CAPT, Vincent. (2012), Écrivainer. La langue morcelée de Samuel Daiber, Lausanne / Golion, Collection de l'art brut / Infolio, coll. « Contre-courant».

Cardinal, Roger et Victor Musgrave. (1979), Outsiders. An Art without Precedent or Tradition, Londres, Arts Council of Great Britain.

DERRIDA, Jacques. (1967), De la grammatologie, Paris, Minuit.

Dubuffet, Jean. (1967), Prospectus et tous écrits suivants, t. 1, Paris, Gallimard.

Foucault, Michel. (1966), Les Mots et les choses, Paris, Gallimard.

JACCARD, Roland. (1975), L'Exil intérieur, Paris, Presses universitaires de France.

LACAN, Jacques. (1966), Écrits, Paris, Seuil.

NANNETTI, colonel astrale, 13 mai- 30 octobre 2011, Lausanne, Collection de l'Art brut.

PEIRY, Lucienne et Sarah Lombardi. (2012), Collection de l'art brut, Lausanne, Skira.

PonGE, Francis. (1961), Le Grand Recueil, t. 2, Paris, Gallimard. 
Queneau, Raymond. (1965), Bâtons, chiffres et lettres, Paris, Gallimard, coll. «Idées ».

QuÉTEL, Claude et Jacques Postel. (2012), Nouvelle Histoire de la psychiatrie, Paris, Dunod.

RORTY, Richard. (1967), The Linguistic Turn. Recent Essays in Philosophical Method, Chicago, University of Chicago Press.

Suberville, Jean. (1948), Théorie de l'art et des genres littéraires, Paris, Éditions de l'École.

THÉvoz, Michel. (1975), L'Art brut, Lausanne, Skira.

-. (1978), Le Langage de la rupture, Paris, Presses universitaires de France.

—. (1979), Écrits bruts, Paris, Presses universitaires de France.

—. (1989), Détournement de l'écriture, Paris, Minuit.

-. (1990), Art brut, psychose et médiumnité, Paris, Éditions de la Différence. 


\title{
Résumé
}

Au sortir de la Seconde Guerre mondiale, Jean Dubuffet nomma par «art brut» des œuvres découvertes en contexte «frontalier»- hôpitaux psychiatriques, campagne reculée, prisons, etc. - et produites par des personnes n'ayant suivi aucun enseignement artistique. Le concept d'« écrits bruts » est apparu dans la filiation de cette conception avant-gardiste de l'art. De par leur origine - majoritairement le contexte asilaire , leur matérialité - graphisme et support non conventionnels -, et le discours qui leur est associé - un éloge de la folie et une critique de la littérature classique-, les «écrits bruts» interrogent de nombreuses limites, et la notion de "frontière » nous est apparue particulièrement riche pour mettre à jour ces différents enjeux.

\begin{abstract}
French painter Jean Dubuffet coined the term "art brut" after the Second World War to designate works of art made in the margins of culture - psychiatric hospital, countryside, and jails and produced by self-taught people. Following from this avantgardist concept of art, the term "écrits bruts" appeared in the seventies to name texts mainly written in mental institutions. Their context of creation - the asylum, their material features unconventional supports and handwritings - and the way they have been interpreted - the creativity of madness as opposed to the inertia of classic literature - question many boundaries. In this essay, we will analyse the notion of "boundary" to unveil the very nature of the "écrits bruts", especially the way they challenge the delimitations of literature and language.
\end{abstract}

